\title{
Germanica
}

\section{Kanaken und andere Schauspieler}

Performative Identitäten bei Feridun Zaimoğlu und Yadé Kara

\section{Julia Bodenburg}

\section{CpenEdition}

\section{Journals}

Édition électronique

URL : http://journals.openedition.org/germanica/418

DOI : 10.4000/germanica.418

ISSN : 2107-0784

Éditeur

Université de Lille

\section{Édition imprimée}

Date de publication : 1 juin 2006

Pagination : 129-140

ISBN : 2-913857-17-5

ISSN : 0984-2632

Référence électronique

Julia Bodenburg, "Kanaken und andere Schauspieler », Germanica [Online], 38 | 2006, Online erschienen am: 19 Februar 2010, abgerufen am 06 Oktober 2020. URL : http://

journals.openedition.org/germanica/418 ; DOI : https://doi.org/10.4000/germanica.418

Ce document a été généré automatiquement le 6 octobre 2020.

(c) Tous droits réservés 


\title{
Kanaken und andere Schauspieler
}

Performative Identitäten bei Feridun Zaimoğlu und Yadé Kara

\author{
Julia Bodenburg
}

\author{
Wir sind allesamt Kanaker, unser Schweiß ist \\ Kanake, unser Leben ist Kanake, unsere \\ Goldketten sind Kanake, unser ganzer eigener Stil \\ ist Kanake. ${ }^{1}<$ Warum trinken Türken TEE? Warum \\ essen sie Oliven zum Frühstück? Warum tragen \\ türkische Frauen ein Kopftuch?» Warum? \\ Warum? Warum? Ist das typisch türkisch? Ist \\ jenes typisch türkisch. Bis du typisch türkisch?2
}

1 Die Literatur von Feridun Zaimoğlu und Yadé Kara ist sicher nicht als «Literatur der Fremde〉, 〈Gastarbeiter- oder Migrantenliteratur〉 zu bezeichnen, und beschreibt man sie als interkulturelle oder türkisch-deutsche Literatur, sitzt man wiederum einem binär ausgerichteten Kulturbegriff auf, der entweder von zwei interagierenden, aber voneinander abgegrenzten Kulturen ausgeht oder von einem integren Ganzen deutscher Literatur, der die Literatur von Minderheiten hierarchisch untergeordnet wird. «Mit Etiketten wie Gastarbeiter-, Ausländer- oder Migrantenliteratur wird freilich immer noch suggeriert, es handele sich um Phänomene, die irgendwie von außen kommen und die entsprechend auch nur aus der Distanz eines gesicherten (Drinnen) sinnvoll betrachtet werden können ${ }^{3}$, so Manuela Günter. Seit ihrer Einschätzung sind einige Jahre vergangen, in denen sich die politischen Debatten um Einwanderung, den möglichen EU-Beitritt der Türkei, «das Kopftuch〉 verdichtet und mit der Forderung einiger Politiker nach einer «deutschen Leitkultur〉 zugespitzt haben. Eine kulturelle Verunsicherung aufgrund von Strukturen der Globalisierung und Pluralisierung wird in Deutschland offensichtlich am liebsten im Zusammenhang mit «den deutschen Türken〉 ausgehandelt. Dass die türkischstämmigen Menschen, die mit 2,5 Mio. die größte Gruppe unter der ausländischen Bevölkerung ausmachen (ca. 600000 von ihnen haben einen deutschen Pass), sich aus der Mitte und von innen heraus artikulieren, evoziert auf deutscher Seite Faszination und Irritation zugleich. So widmete DIE ZEIT beispielsweise (den Türken) eine thematische Serie und Georg Dietz titelte in der Frankfurter Allgemeinen Sonntagszeitung «Der Halbmond ist aufgegangen. Avantgarde der 
Widersprüche: Warum die deutschen Türken nicht nur unsere Wirtschaft retten können, sondern auch unsere Kultur» ${ }^{4}$.

2 Die türkischstämmigen Schriftsteller Feridun Zaimoğlu und Yadé Kara können als zwei Vertreter der mit Faszination und Verwunderung beäugten Gruppe gelten, die eine kulturelle Differenz ‘von innen〉 in das Medium der «deutschen〉 Literatur hineintragen und damit die Vorstellung eines homogenen Ganzen in Frage stellen oder direkt angreifen. Feridun Zaimoğlu wurde $1964 \mathrm{im}$ türkischen Bolu geboren und lebt seit über 30 Jahren mit kurzen Unterbrechungen in Deutschland, zuletzt in Kiel. Yadé Kara, Jahrgang 1965, wurde ebenfalls in der Türkei, in Cayirli, geboren, arbeitete in Istanbul, Berlin, London und Hongkong und lebt in Berlin. Ihre auf Deutsch verfassten Texte lassen sich nicht als Literatur von Migranten bezeichnen, die von außen kommen und sich an den Rändern einer vermeintlich deutschen Kultur verorten, sondern thematisieren auf ironische Weise selbst den vom deutschen Literaturbetrieb als Problem aufgefassten Bezeichnungstrubel und die mitgeführten Kultur- und Identitätskonzepte ${ }^{5}$. Es wurde zumindest in Bezug auf Zaimoğlus Texte (ich gehe im Folgenden auf seine frühen Texte Kanak Sprak, 1995?; Abschaum, 1997?; Koppstoff, 1998und auf den 2002 erschienenen Roman German Amok ein) herausgearbeitet, dass sie sich eben diesen Beschreibungskategorien verweigern, indem sie dichotome Grenzsetzungen, die für die Einordnung von deutschsprachiger, aber «nicht ganz deutscher> Literatur konstruiert und herangezogen werden, unterlaufen ${ }^{6}$. Die hier in den Blick genommenen Texte von Zaimoğlu und Kara sind einer veralteten, nationalisierenden Auffassung von Kultur und damit einhergehenden Konzeption einer starren, in ethnischen Wurzeln verankerten Identität, wie sie von einigen deutschen Politikern und Kulturschaffenden mittels der (Kopftuch)- oder (Leitkultur)-Debatte vertreten wird, ein großes Stück voraus. Ich gehe im Folgenden auf die narrativen und ästhetischen Strategien ein, die die Texte in dieser Hinsicht einsetzen und versuche, sie vor dem Hintergrund aktueller kulturpolitischer Debatten zu situieren?

\section{Feridun Zaimoğlus Kanak Attacken}

3 Seit Mitte der 90er Jahre inszeniert sich Feridun Zaimoğlu als Wortführer für TürkischDeutsche und andere -Deutsche, die in der öffentlichen Mediendiskussion bis dahin kaum sichtbar waren. Er attackiert vor allem in seinen ersten drei Büchern Kanak Sprak (1995), Abschaum (1997) und Koppstoff (1998), die aus 〈Interviews〉 mit jungen Kanakstern (eine Wortschöpfung aus Kanake, Youngster und Gangster) bestehen, eine deutsche Politik der Multikulturalität, die die sog. Gastarbeiter- oder Migrantenliteratur staatlich fördert und gleichzeitig als «Literatur am/des Rand/s» ausgrenzt.

«Hier hat allein der Kanake das Wort» ${ }^{8}$. Feridun Zaimoğlu verspricht in Kanak Sprak ein «authentisches〉 Sprachbild» ${ }^{9}$ von türkischen «Gastarbeiterkindern〉 der zweiten und dritten Generation. Mit der vermeintlichen Authentizität der 〈Interviews〉, die Zaimoğlu als das Produkt «detektivischer» Nachforschungen im «Milieu», im Kiez der Männer» ${ }^{10}$ ausgibt, spricht er eine Kategorie an, die die «Betroffenhetsliteratur» türkischstämmiger Autoren und Autorinnen seit den 70er Jahren begleitet hat. Den «aarmen, aber herzensguten Türken Ali»» jener «(Müllkutscher-Prosa»» ${ }^{11}$ wird man in Zaimoğlus (Protokollen〉 ebenso wenig antreffen wie eine authentische Abschrift der 〈Interviews`, die er mit den Kanaken geführt zu haben erklärt. Um nämlich der 
«Folklore-Falle»12 $\mathrm{zu}$ entgehen, musste Zaimoğlu den Jargon der Kanaken übersetzen und weitreichende Eingriffe vornehmen. Die Authentizität seiner «Nachdichtung» ${ }^{13}$ ist also fingiert und damit wird auch die Programmatik des gesamten Projekts der Kanak Sprak $^{14}$ einerseits und andererseits die Konstrukthaftigkeit der typisierten Kanaken (z.B. «der Zuhälter», «der Islamist», «der Kleinhehler»), die in dem Buch zu Wort kommen, ausgestellt.

5 Kanak Sprak ist eine Kunstsprache, die sich aus so unterschiedlichen Quellen wie Jugendslang, englischsprachigem Hip-Hop-Vokabular, literarischer Hochsprache, norddeutschem Dialekt, rotwelschen und jiddischen Bruchstücken speist. Mit Kanak Sprak ist also nicht einfach der Straßenjargon («Ey Alder, das is krass» u.ä.) von Jugendlichen jedweder ethnischer Herkunft gemeint, sondern «ein künstliches Idiom, das sich zu seiner Künstlichkeit bekennt» ${ }^{15}$. Nun war die Bezeichnung 〈Kanake〉 lange Zeit eine diskriminierende Benennung vorwiegend für Deutsche türkischer Abstammung. 〈Kanak〉 bedeutet im Hawaianischen 〈Mensch〉; in der Kolonialzeit übernahmen Europäer diesen Begriff für die abwertende Bezeichnung von Südseeinsulanern. Mit Bezug auf die positive Umwertung des eigentlich von Weißen verwendeten, diskriminierenden Begriffs «Nigger`, führt Zaimoğlu das Wort 〈Kanake〉 als stolze Selbstbezeichnung der (Deutschtürken) an, und er tut das mit einer Wucht, die ihn Mitte der 90er Jahre zum Sprachrohr für eine ganze Bevölkerungsgruppe hat werden lassen. Die Umwertung funktioniert dabei auf ganz ähnliche Weise, wie die amerikanische Philosophin Judith Butler es für hate speech beschrieben hat. ${ }^{16}$ Butler hatte J.L. Austins Sprechakttheorie insofern politisch erweitert, als sie zum einen auf den Zusammenhang von Bezeichnungspraxis und der Artikulation von Identität und zum anderen auf die Beschaffenheit des Sprechakts als körperliche Handlung hingewiesen hat, eine Handlung, die mittels einer Anrufung durch den Anderen verletzen kann:

Die Anrufung ist ein Sprechakt, dessen «Inhalt〉 weder wahr noch falsch ist, weil ihre erste Aufgabe gar nicht in der Beschreibung besteht. Ihre Absicht ist es vielmehr, ein Subjekt in der Unterwerfung zu zeigen und einzusetzen, sowie seine gesellschaftlichen Umrisse in Raum und Zeit hervorzubringen. Ihr reiteratives Verfahren hat den Effekt, ihre 〈Position) über die Zeit hinweg zu sedimentieren» ${ }^{17}$.

6 Bezeichnen können nur diejenigen, die bereits benannt sind. Wenn also jemand, der schon als 〈Deutscher» bezeichnet worden ist, 〈den Türken〉 als 〈Kanake〉 benennt, erschafft er ihn im Augenblick der Anrufung und weist ihm bestimmte Bilder zu. Diese Position findet sich in Zaimoğlus Texten wieder: «Der Aleman ist $\mathrm{n}$ wilder Exotenmaler in seinem Element, und er erschafft uns mit seiner Kleckswut-Kunst». ${ }^{18}$ In einer solchen Konzeption wird Identität nicht als etwas Gegebenes gedacht, sondern als performativ erzeugte, und sie kann sich nur, wie es der britische Kulturwissenschaftler Stuart Hall ausgeführt hat, innerhalb der Vor-bilder vollziehen, d.h. innerhalb der Zuschreibungen, mit denen die Angerufenen benannt und damit konstituiert werden: «Statt Identität als eine schon vollendete Tatsache zu begreifen, die erst danach durch neue kulturelle Praktiken repräsentiert wird, sollten wir uns vielleicht Identität als eine (Produktion) vorstellen, die niemals vollendet ist, sich immer in einem Prozeß befindet, und immer innerhalb - nicht außerhalb - der Repräsentation konstituiert» ${ }^{19}$. Die der Performanz inhärente Struktur der Wiederholung bietet laut Butler aber auch die Möglichkeit des Widerstands. Durch eine gezielte, positive Fehlaneignung kann verletzende Sprache umdirigiert werden ${ }^{20}$ : Zaimoğlus Kanaken entwenden den Begriff 〈Kanake〉 dem rassistischen Diskurs, sie dekontextualisieren ihn und eignen ihn sich als 
〈richtigen〉 Namen an. «Kanake! Dieses verunglimpfende Hetzwort wird zum Identität stiftenden Kennwort, zur verbindenden Klammer dieser (Lumpenethnier»»" ${ }^{21}$. In der subversiven Resignifizierung wird das Schimpfwort gegen die Aggressoren gewendet. So beschreibt es auch Ertan Ongun, der Protagonist in dem 1997 erschienenen Buch Abschaum, das auch die literarische Vorlage für den Spielfilm Kanak Attack lieferte: «Wir sind die Kanaken, vor denen ihr Deutschen immer gewarnt habt. Jetzt gibt es uns, ganz eurem Bild und euren Ängsten entsprechend ${ }^{22}$. Zaimoğlus Kanak Sprak kann also als verstörende Widerrede im Sinne Butlers beschrieben werden, verstörend deshalb, weil ihre Sprecher den Aggressoren das entgegen schleudern, mit dem diese versucht haben, «die Türken` als gefährliche und daher als zu kontrollierende Marginalität zu diffamieren. Der sich selbst als 〈Kanake〉 Bezeichnende entzieht sich der Definitionsmacht des herrschenden Diskurses und greift die brüchige Trennung zwischen 'wir Deutschen〉 und (ihr Türken〉 an. ${ }^{23}$ Butler nennt dies «postsouveräne Handlungsmacht ${ }^{24}$, allerdings nicht ohne darauf hinzuweisen, dass die Widerrede immer Spuren der «einstigen` Diskriminierung in sich trägt.

Die körperliche Dimension des performativen Sprechakts, die Butler hervorhebt ${ }^{25}$, betont auch Zaimoğlu in seinem Vorwort zu Kanak Sprak:

«Er [der Kanake] unterstreicht und begleitet seinen freien Vortrag mimisch und gestisch. Die reiche Gebärdensprache des Kanaken geht dabei von einer Grundpose aus, der so genannten (Ankerstellung): Die weit ausholenden Arme, das geerdete linke Standbein und das mit der Schuhspitze scharrende rechte Spielbein bedeuten dem Gegenüber, daß der Kanake in diesem Augenblick auf eine rege Unterhaltung großen Wert legt» ${ }^{26}$.

Kanak Sprak ist auf vielfache Weise mehrsprachig. Die Figur des Kanaken misst durch ihre äußerst lustvolle Körpersprache das Territorium aus, auf dem sie sich befindet, und zwar performativ; ihr Handlungsraum entsteht erst durch die Bewegung des Beschreibens: «Und über die einzelne charakteristische Gebärde hinaus signalisiert der Kanake: Hier stehe ich und gebe mit allem, was ich bin, zu verstehen: Ich zeige und erzeuge Präsenz» ${ }^{27}$. Jener reichen Gebärdensprache eignet ebenfalls die Struktur der Wiederholung, denn nur so kann sie zum Erkennungsmerkmal des Kanaken und zur Identität stiftenden Pose werden, die angeeignet werden kann. Die Erzähler und Erzählerinnen in Zaimoğlus Kanak Sprak-Bänden erzeugen ihre Identität, indem sie sich mit ihrem ganzen Körper zur Schau stellen, sich auf die selbst ausgemessene Bühne bringen und auf diese Weise das Gebot des «Alemannen`, das Sprechen vom Körper zu trennen ${ }^{28}$, programmatisch überschreiten.

9 Das Moment des Inszenatorischen ist für die Identifikation unabdinglich und wird von den anderen, das sind hier die Angehörigen der Mehrheitsgesellschaft, auch immer wieder eingefordert - allerdings lautet die Forderung dann: 〈Sei Türke!〉. Von den (Dichter-Figuren〉 in Zaimoğlus Texten wird die zwanghafte Aufforderung, sich als (türkischer) Migrant, als Anderer eindeutig und ein für alle Mal zu identifizieren, jedoch als unzumutbar zurückgewiesen ${ }^{29}$. "Zaimoğlu konstruiert seine Kanaken polyvok und dissident $»^{30}$. Sie entgehen dem 〈alemanischen〉 Versuch, Differenzen aufzustellen, die für die eigene Identität konstitutiv sind, indem er die ethnische Identifizierung unkenntlich macht. Manuela Günter resümiert:

Nicht Kulturen treffen im «Kanaken` aufeinander, sondern er ist selbst das Resultat einer bereits hybriden Kultur aus türkischen, deutschen, regionalen, religiösen, alters- und geschlechtsspezifischen Elementen, die Identität nur als stets wechselnde Posen denken lässt und die offensichtlich durch ihre Uneindeutigkeit 
bei der Gegenseite immer wieder das Bedürfnis nach einer klaren Markierung provoziert $^{31}$.

\section{¿Deutsch-deutsche〉 Geschichte: Ossi- und Wessitürken in Yadé Karas Selam Berlin}

Eine solch hybride Szenerie entwirft auch Kara in ihrem 2003 erschienenen Debüt. Die Romanhandlung umfasst die Zeit vom Fall der Berliner Mauer bis zum Tag der Wiedervereinigung und nimmt damit augenscheinlich das «deutsche〉 Thema schlechthin in den Blick. Nur ist das vermeintlich «Deutsche» hier mehrfach differenziert und vermischt und gar nicht mehr so 〈deutsch〉: Hasan, der 19jährige IchErzähler, Sohn eines türkischen Ingenieurs, der in den 70er Jahren nach Deutschland emigriert war, begreift sich als Kreuzberger mit «Nomadenblut». Die deutsch-deutsche Gespaltenheit in 〈Wessis〉 und 〈Ossis〉 entpuppt sich als mehrschichtige Differenziertheit. Als Hasan nämlich erfährt, dass sein Vater aus einer Affäre mit einer ostdeutschen Frau einen weiteren Sohn hat, wird dieser als «Ossitürke», als die andere Version der «Wessitürkin» Leyla, Hasans Cousine, eingeführt ${ }^{32}$. Der Roman ist mit weiteren transdifferentiellen Figuren ausgestattet. Neben Hasans türkisch-deutschen Freunden gibt es z.B. Cora, deren irakischer Vater in Amerika lebt, während sie und ihre Mutter über Ungarn von Ostberlin nach Westberlin flüchteten, oder die Inderin Sukjeet, die in London lebt und ein Englisch «wie die Queen» spricht ${ }^{33}$. Das Berlin der Wende kann als Matrix eines offenen deutschen und gleichsam europäischen Transitraums gelesen werden, dessen Teilungen unterschiedlichen kulturellen Differenzen Raum geben.

11 Hasan setzt seine kulturelle Differenz spielerisch und strategisch ein. Um eine kleine Filmrolle zu bekommen, ist er (ganz Kanake): «Ich hatte gleich gecheckt, was Wolf suchte. Er suchte so einen Spielothekentürken mit Cowboystiefeln. Und den spielte ich ihm jetzt vor $\aleph^{34}$. Er bestätigt zwar sein Vor-Bild - mit Berliner Akzent - weiß aber, was er tut und dass er es tut, er reflektiert die Konstrukthaftigkeit kultureller Zuschreibungen. Allerdings verbleibt diese reflektierte Position Wolf gegenüber im Verborgenen: «Erstens Mann, zweitens Ehre, drittens Waffen - das ist doch typisch bei euch?〉 Ich nickte. (Interessante kulturelle Eigenart...), lächelte Wolf. Ich nickte weiterhin. Seine blauen Augen trübten sich rot. Der Rotwein wirkte» ${ }^{35}$. Die politische Sprengkraft einer Kanak Attacke, wie Zaimoğlu sie in seinen Episoden inszeniert hat, die, metaphorisch gesprochen wie Gewehrsalven daherkommen, geht hier zwar nicht verloren, ist jedoch abgeschwächt. Die Figur des Hasan ist zu sehr als pikaresker Jugendlicher auf der Suche nach Job, Frau, Coolness gestaltet und hat eine Geschichte zu erzählen. Die Momente, in denen er als verstörender Kanake auftritt, sind daher ungleich beiläufiger innerhalb der Mauerfall-Handlung gestaltet, wie z.B. eine Szene am Filmset, in der Hasan, Erbsensuppe mit Wurst essend, auf die deutsche Margit trifft:

«Moslem und Schweinewurscht. Geht des denn?〉 blökte sie. [...] «Bisch doch Tüürkee...und Moslem...oda?〉 [...] 〈Wohär kommsch du eigentlich?〉〈Aus Kreuzberg...Aber», sagte ich kurz. «Wirklich du bisch gar net wie die andern Türken...〉 [...] «Neee, du siehsch mehr italienisch-spanisch aus. Die Türken sind klein und dick und haben Kopftuch. [...] Verschtehsch du eigentlich gut Deutsch?> Und sie blickte mit Ekel auf meine Wurst. Ich verschlang den Rest und wischte mir mit dem Ärmel über den Mund. «Manieren hat ma in deiner Heimat au net, oda...?〉 [...] «Bei uns in Kreuzberg heißt es : Friß oder stirb!〉 Ich drückte ihr den leeren 
Suppenteller in die Hand und ging. Ich glaube, sie stand noch stundenlang da und schaute verdutzt hinter mir her ${ }^{36}$.

Hasans Identitätskonzept kann als Rolle beschrieben werden. Sein Schauspiel und die alltäglichen Performances setzt er strategisch ein, nicht immer mit Erfolg. Denn obwohl der Text recht unkritisch das ganze Register postkolonialer Theorie herunterbetet, versäumt er es an einigen wenigen Stellen nicht, auf die ungleichen Bedingungen der Identitätsbildung hinzuweisen. Kara legt ihr Augenmerk beispielsweise auf die assymmetrische Konstellation zwischen dem Protagonisten und dem Regisseur und macht so auf die Machtverhältnisse aufmerksam. ¿Der Türke〉 Hasan ist für Wolf lediglich eine Figur, und Hasan ist überzeugt, dass er diese Mauer in Wolfs Kopf nicht einreißen kann: «Die Bilder in seinem Kopf sind wie in Beton gegossen. Dieser Macker benutzte mich nur dazu, seine abgewichste Meinung, seine Bilder, Vorstellungen zu bestätigen. Schließlich war er der Regisseur, er hatte Ahnung, er hatte recht. Punkt. Weiter nichts! ${ }^{37}$. Wolf konsumiert kulturelle Stereotype als das Andere, das er braucht, im sich der eigenen Identität zu versichern. Seine vielfältigen Rollen kann Hasan nur innerhalb des bereits Repräsentierten spielen. Dass die Mittel der Identitätsbildung ungleich verteilt sind, zeigt der Roman zwar, er bietet aber keine Strategien an, um dieses assymmetrische Verhältnis zu verändern, kurz, Hasan ist keine subversive Figur ${ }^{38}$.

Ich möchte noch einmal kurz auf den anfangs angesprochenen Aspekt der «deutschdeutschen〉 Geschichte, die hier aus türkisch-deutscher Perspektive erzählt wird, zurückkommen. Bei aller zumeist etwas platt wirkenden popliterarischen Ausrichtung des «Berlinromans〉 ist ihm zu Gute zu halten, dass er ein anderes, vielseitigeres Bild der Metropole zum Zeitpunkt des Mauerfalls entwirft, als es in vielen westdeutschen Köpfen besteht. Die Ossis und Wessis, die hier aufeinander treffen, sind längst um ein Vielfaches ausdifferenziert. Berlin ist ein Bedeutungsraum, in dem Positionierungen und Grenzen ständig gegeneinander verschoben werden. Diese nicht erst seit der Wiedervereinigung bestehende Transkulturalität sollte in gerade wieder aufkommenden 〈Leitkultur`-Debatten berücksichtigt und adäquat repräsentiert werden ${ }^{39}$.

\section{Differenzen im Leerlauf : Feridun Zaimoğlus German Amok}

14 Zaimoğlus 2002 erschienener Roman German Amok, auf den ich in aller Kürze zum Schluss eingehen möchte, knüpft an das transkulturelle Berlin an. Während es bei Kara noch als aufstrebende, von Differenzen profitierende und kreative Metropole gefeiert wurde, wird es bei Zaimoğlu als Unort, als von innen zerfressener, an sich selbst zu Grunde gehender Kollektivkörper entworfen. Schauplatz in German Amok ist eine dekadente Kunstschickeria in Berlin, «dieser für immer verfluchten und vergammelnden Metropole» ${ }^{40}$. Der türkischstämmige Ich-Erzähler, ein erfolgloser Maler, nimmt mit Abscheu und voyeuristischer Lust an den Ritualen der Kunstszene teil, von der er finanziell abhängig ist. Er nährt sich wie ein «Parasit» ${ }^{41}$ von seinen Mitmenschen, die er allesamt als verachtenswürdige Kreaturen beschreibt, und von einer übersättigten, abgestumpften Gesellschaftsschicht. Die Szenerie ist in Transdifferenzen aufgespalten: quer zu der Differenz «türkisch-deutsch〉 verlaufen vielfältige weitere Differenzen, z.B. gibt es neben dem türkischen Hodscha mit 
Gentlemanbart die japanische Tanzlehrerin, die lieber ein Exotenenglisch spricht, weil sich Deutsch unter Künstlern keiner Beliebtheit erfreut und das Alte Testament bemüht, wenn sie fluchen will ${ }^{42}$.

Der in pluralistische Differenzen aufgesprengten Metropole wird das provinzielle Niemandsland, Treptin in Brandenburg, gegenübergestellt. «Das hier ist ein verdammtes Entwicklungsland, nein, schlimmer noch, wir sind im Busch» ${ }^{43}$. Indem der Erzähler den Osten Deutschlands als kolonialen, unzivilisierten Raum abwertet, stellt er die Fremdbilder aus, die eigentlich auf ihn selbst gerichtet sind. Diese Aneignungsgeste diskriminierender Fremdbilder kann jedoch weniger als positive Fehlaneignung im Sinne Butlers gelesen werden, da hier überhaupt keine Subjektpositionen mehr zur Verfügung stehen: Der Titel German Amok deutet an, dass diese aufgelöst sind. Läuft jemand Amok, befindet er sich in einem Ausnahmezustand, ist unzurechnungsfähig, läuft gewissermaßen im Leerlauf. Im Roman sind deshalb keine Positionen feststellbar und einnehmbar, weil alle Differenzen durch ihre eigene Hyperbolik überboten werden. Die Metapher des Parasiten zeigt außerdem an, dass der «deutsche Volkskörper〉 schon von innen zerfressen ist und sich selbst zerstört. Hat sich die Bezugsgröße «des Deutschen〉 selbst schon bis zur Unkenntlichkeit ausdifferenziert, kann auch «das Andere» nicht mehr verortet werden. Der Text bewegt sich damit jenseits von Konzepten der Transkulturalität oder Transdifferentialität und setzt dem Gedanken eines positiven Potentials kultureller Vermischung ein Szenario von Degeneration und Tabulosigkeit entgegen.

Gerade in Hinblick auf Feridun Zaimoğlus Werkentwicklung kann German Amok als kritischer Text gelesen werden, der genau das in Szene setzt und zynisch überbietet, was Tom Cheeseman 〈Kanak-Chic〉 nennt ${ }^{44}$. Er meint damit eine Popularisierung, Kommerzialisierung und Vereinnahmung des rebellischen (Kanaken〉, was die Entpolitisierung der Kategorie 〈Kanak〉 zur Folge hat ${ }^{45}$. Zaimoğlu selbst spielt dieses Spiel gewissermaßen mit und lässt sich doch nicht einordnen. Die Bilder, die für ihn vorgesehen sind, sei es der «Kieler Salontürke» ${ }^{46}$ oder der «Malcolm X der Türken» ${ }^{47}$, erfüllt er, um sie im nächsten Moment wieder zu dekonstruieren und sich so eines an ihn gerichteten Bedürfnisses nach eindeutiger Positionierung zu entziehen.

In Zaimoğlus German Amok und auch in Karas Selam Berlin lassen sich bei den Figuren Tendenzen einer großen Müdigkeit hinsichtlich der beständigen Forderung, sich als 〈Türke〉 bzw. 〈Türkin` zu erklären, feststellen. Zaimoğlu begegnet dieser Forderung mit Provokation, indem er den «deutschen Volkskörper» wie auch seine «Bewohner» als verfault und degeneriert beschreibt. Kara geht einen anderen Weg. In ihrem Poproman werden theoretische Konzepte (Hybridität, Transkulturalität), die für die Untersuchung jener «nicht ganz deutschen» Literatur als Analyseinstrumentarium herangezogen wurden, platt und unkommentiert heruntergebetet. Beide Gesten können m. E. als Versuch eines Rückzugs aus den Mechanismen eines in Deutschland vermeintlich praktizierten Multikulturalismus gelesen werden, der sich bei genauerem Hinsehen als Stabilisierung hierarchischer Verhältnisse zwischen deutschem Zentrum und türkischer Marginalität entpuppt. 


\section{NOTES}

1. Ertan Ongun in Lars Beckers Film Kanak Attack, Deutschland 2000.

2. Yadé Kara : Selam Berlin, Zürich, Diogenes, 2003, S. 204.

3. Manuela Günter : «(Wir sind bastarde, freund...) Feridun Zaimoglus Kanak Sprak und die performative Struktur von Identität», in : Sprache und Literatur, 1999, Bd. 83, S. 15-27, S. 15.

4. Vgl. die ZEIT-Serie «Türken in Deutschland», DIE ZEIT Nr. 35-37 (August/September 2003). Georg Dietz: «Der Halbmond ist aufgegangen. Avantgarde der Widersprüche: Warum die deutschen Türken nicht nur unsere Wirtschaft retten können, sondern auch unsere Kultur», Frankfurter Allgemeine Sonntagszeitung Nr. 24 (22. Juni 2003), S. 19.

5. Vgl. Sabine Keiner : «Von der Gastarbeiterliteratur zur Migranten- und Migrationsliteratur literaturwissenschaftliche Kategorien in der Krise?», in: Sprache und Literatur, 1999, Bd. 83, S. 2-14. Beide Autoren sind außerdem mehrfach ausgezeichnet worden - Zaimoğlu z.B. mit dem Preis der Jury beim Ingeborg Bachmann-Wettbewerb 2003 und 2005 mit dem Adalbert-vonChamisso-Preis, Kara 2004 mit dem Deutschen Bücherpreis in der Kategorie «Erfolgreiches Debüt» und mit dem Adalbert-von-Chamisso-Preis - und damit auch offiziell im deutschen Literaturbetrieb vertreten.

6. Vgl. die folgenden Aufsätze : Manuel Günter : «(Wir sind bastarde, freund...»» a.a.o.?; Kati Röttger : «Wie werde ich Ausländer?» Kanak Sprak, Sprechen lernen und performative Strategien der Entfernung», in : Inszenierungen: Theorie - Ästhetik - Medialität, hg. v. Christopher Balme und Jürgen Schläder, Stuttgart/Weimar, Metzler, 2002, S. 117-134?; überblicksartig zu Zaimoğlu : Tom Cheeseman : "Akçam - Zaimoğlu - ,Kanak Attak»: Turkish Lives and Letters in German», in : German Life and Letters, 2002, Bd. 55:2, S. 180-195.

7. Der vorliegende Aufsatz hat sich aus dem Forschungsprojekt «Antipodean Visions of Transcultural Societies», in dem ich unter der Leitung von Martina Wagner-Egelhaaf seit 2001 mit arbeite, ergeben. Teile des Aufsatzes werden in leicht veränderter Form in dem Buch von Hendrik Blumentrath, Julia Bodenburg, Roger Hillman, Martina Wagner-Egelhaaf: Münster, Aschendorff, 2006 [in Vorbereitung] veröffentlicht. Ich danke den Projektteilnehmern für die anregende, gemeinsame Arbeit.

8. Feridun Zaimoğlu: Kanak Sprak. 24 Mißtöne vom Rande der Gesellschaft, Hamburg, Rotbuch, 1995,

S. 18.

9. Ebd., S. 18.

10. Ebd., S. 15.Im ersten Kanak Sprak-Band von 1995 kommen nur männliche Kanaken «zu Wort»; 1998 erschien ein zweiter Band, der diesmal ausschließlich weibliche Kanakas in den Blick nimmt. Wo im Folgenden von 〈dem Kanaken〉 die Rede ist, ist demnach auch nur der männliche gemeint.

11. Ebd., S. 12.

12. Ebd., S. 14.

13. Ebd.

14. Feridun Zaimoğlu ist auch Mitbegründer von Kanak Attak, einem 1998 «selbstgewählten Zusammenschluß verschiedener Leute über die Grenzen zugeschriebener, quasi in mit in die Wiege gelegter 〈Identitäten〉 hinweg.» In seinem Manifest wendet sich Kanak Attak gegen kulturellen Rassismus und die Befürworter eines (Mültikültüralizm), der sog. Nichtdeutsche aufgrund des ihnen zugeschriebenen Exotismus vereinnahmt. «Kanak Attak bietet eine Plattform für Kanaken aus den verschiedensten gesellschaftlichen Bereichen, denen die Leier vom Leben zwischen zwei Stühlen zum Hals raushängt». http://www.kanak-attak.de/ka/about/ manif_deu.html (Stand 03.01.06) 
15. Monika Schmitz-Emans: «(Die Wortgewalt des Kanaken». Formen und Funktionen literarischer Mehrsprachigkeit», in: IABLIS, 2002, Bd.1: Migration: Die Erzeugung von Zwischenwelten, abzurufen unter http://www.iablis.de/iablis t/2002/schitz-emans.htm (Stand 03.01.06).

16. Vgl. Judith Butler: Haß spricht. Zur Politik des Performativen, Berlin, Berlin Verlag, 1998.

17. Ebd., S. 54 .

18. Feridun Zaimoğlu: Koppstoff. Kanaka Sprak vom Rande der Gesellschaft, Hamburg, Rotbuch, 1998, S. 85. Vgl. Feridun Zaimoğlu: Kanak Sprak, a.a.O., S. 31-32.

19. Stuart Hall: «Kulturelle Identität und Diaspora», in: S.H.: Rassismus und kulturelle Identität, übers. u. hg. v. Ulrich Mehlem, Hamburg, Argument, 1994, S. 26-44, S. 26.

20. Vgl. Judith Butler: Haß spricht, a.a.O., S. 64.

21. Feridun Zaimoğlu: Kanak Sprak, a.a.O, S. 17.

22. Feridun Zaimoğlu: Abschaum, a.a.O., S. 183.

23. Vgl. dazu Kati Röttger: «(Wie werde ich Auslänger?»», a.a.O., S. 126.

24. Vgl. Judith Butler: Psyche der Macht. Das Subjekt der Unterwerfung, Frankfurt a. M., Suhrkamp, 2001, bes. S. 16-22.

25. In Körper von Gewicht ist es Butlers Unterfangen, die Frage nach der Materialität des Körpers mit der Performativität der sozialen Geschlechtsidentität zu verknüpfen. Sie versteht unter Performativität «die ständig wiederholende und zitierende Praxis, durch die der Diskurs die Wirkungen erzeugt, die er benennt. » Es seien «die regulierenden Normen des biologischen Geschlechts», die in performativer Wirkungsweise die Materialität des Körpers konstituieren». Judith Butler: Körper von Gewicht. Die diskursiven Grenzen des Geschlechts, Frankfurt a.M., Suhrkamp, 1995, S. 22.

26. Feridun Zaimoğlu: Kanak Sprak, a.a.O, S. 13.

27. Ebd., S. 14.

28. «Das Schlimme an dieser Verständigung ist, daß man beim Reden das Gesicht totstellen muß und die Hände ruhig halten». Feridun Zaimoğlu: Koppstoff, a.a.O., S. 91.

29. Vgl. Feridun Zaimoğlu: Kanak Sprak, a.a.O., S. 84-86.

30. Manuela Günter: «〈Wir sind bastarde, freund...»», a.a.O., S. 22.

31. Ebd., S. 27.

32. Yadé Kara: Selam Berlin, a.a.O., S. 252.

33. Ebd., S. 261.

34. Ebd., S. 222.

35. Ebd., S. 245.

36. Ebd., S. 254-255.

37. Ebd., S. 245.

38. Vgl. zur Kritik an der deutschsprachigen Rezeption der Kategorie der Hybridität Mark Terkessidis: «Globale Kultur in Deutschland oder: Wie unterdrückte Frauen und Kriminelle die Hybridität retten», in: parapluie, 1999, Bd. 6, http://parapluie.de/archiv/generation/hybrid/ (Stand 03.01.06).

39. Der großangelegten Medienkampagne «Du bist Deutschland», die von September 05 bis Januar 06 im deutschen TV, Kino und als Printmedium lief, gelang das tatsächlich ganz gut: Das Angebot der identifikatorischen Leitfiguren reichte von Günther Jauch, Xavier Naidoo, bekannten Gesichtern aus Fernsehen, z.B. dem am Down-Syndrom leidenden Schauspieler Bobby Brederlow über Gerald Asamoah, Minh-Khai Phan Thi über Kool Savas bis Anne Will und Marcel Reich-Ranicki. Vgl. www.du-bist-deutschland.de (Stand 03.01.06).

40. Feridun Zaimoğlu: German Amok, Köln, Kiepenheuer \& Witsch, 2002, S. 53.

41. Vgl. ebd., S. 44, S. 192.

42. Vgl. ebd., S. 118.

43. Ebd., S. 134. 
44. Vgl. Tom Cheesman: «Akçam - Zaimoğlu - «Kanak Attak»: Turkish Lives and Letters in German», a.a.O., S. 180.

45. Vgl. ebd., S. 193.

46. So bezeichnete der Satiriker Wiglaf Droste ihn, zitiert nach Tom Cheeseman: «Akçam Zaimoğlu - 〈Kanak Attak»», a.a.O., S. 194.

47. Vgl. diese und andere Zuschreibungen auf der Internetseite http://www.singlegeneration.de/kohorten/feridun Zaimoğlu.htm (Stand 03.01.06).

\section{RÉSUMÉS}

Die in der Türkei geborenen, in Deutschland lebenden und in deutscher Sprache schreibenden Schriftsteller Feridun Zaimoğlu und Yadé Kara gehören $\mathrm{zu}$ den Vertretern einer neuen deutschsprachigen Literatur, die mit den Ordnungsbegriffen einer ,Migranten- oder Ausländerliteratur' nicht mehr zu fassen ist. Karas und Zaimoğlus Figuren artikulieren sich aus der Mitte der 〈deutschen〉 Gesellschaft heraus und stellen vor Augen, dass diese längst vielfach differenziert ist. (Deutsche) Räume, wie z.B. das Berlin der Wende, werden als transkulturelle Bedeutungsräume entworfen, in denen Positionierungen keineswegs stabil sind, sondern von den Subjekten immer wieder verschoben oder neu 〈erspielt〉 werden müssen. Das Moment des Inszenatorischen spielt dabei eine herausragende Rolle: Identität erscheint als Performance und Schauspiel. Im vorliegenden Beitrag werden ausgewählte Texte von Feridun Zaimoğlu (Kanak Sprak, 1995; Abschaum, 1997; Koppstoff, 1998; German Amok, 2002) und Yadé Karas Debütroman Selam Berlin (2003) auf die Fragestellung hin untersucht, wie Identität in einer von vielfältigen kulturellen Vermischungen und Differenzen geprägten Gesellschaft entworfen wird.

Les écrivains Feridun Zaimoğlu et Yadé Kara nés en Turquie, vivant en Allemagne et écrivant en allemand, comptent parmi les représentants d'une nouvelle littérature de langue allemande qu'on doit cesser d'identifier exclusivement comme une «littérature de migrants ou d'étrangers ». Les personnages de Kara et de Zaimoğlu font partie intégrante de la société 'allemande' qui présente depuis longtemps de multiples facettes. Des espaces 'allemands', comme par exemple le Berlin de l'unification allemande, sont présentés comme des espaces de sens transculturels dans lesquels les positions des personnages ne sont jamais fixées une fois pour toutes, mais, au contraire, où ceux-ci habitent ces espaces et les remettent constamment en jeu. D'où le rôle éminemment important de la mise en scène : l'identité apparaît comme mise en scène et spectacle. À la lumière d'un choix de textes de Feridun Zaimoglu (Kanak sprak, 1995?; Abschaum, 1997?; Koppstoff, 1998?; German Amok, 2002)ainsi que du premier roman de Yadé Kara (Selam Berlin, 2003), nous étudierons dans cette contribution comment est traitée la question de l'identité dans une société marquée par une grande diversité culturelle.

\section{INDEX}

Mots-clés : Turquie, émigration turque, littérature de la migration, Berlin, identité oeuvrecitee Kanak sprak, Abschaum, Koppstoff, German Amok, Selam Berlin 
AUTEURS

JULIA BODENBURG

Westfälische Wilhelms-Universität Münster 